\title{
Genome-wide identification and functional characterization of CDPK gene family reveal their involvement in response to drought stress in Gossypium barbadense
}

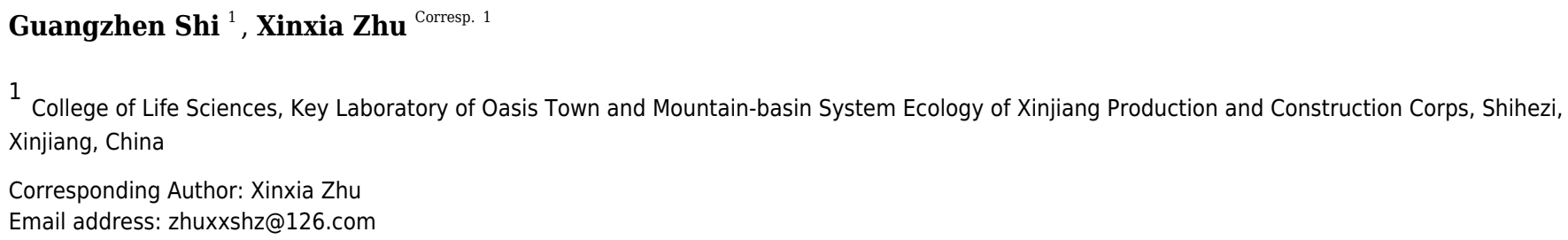

Background: Calcium dependent protein kinases (CDPKs) are a class of important calcium signal sensing response proteins, which play an important regulatory role in response to abiotic stress. However, researchers have not been excavated CDPKs' role in drought in sea-island cotton(Gossypium barbadense L. 'H7124'). Results: 84 CDPK genes have been identified in G. barbadense. These GbCDPK genes are unevenly distributed on 26 chromosomes, and segmental duplication is the significant way for the extension of CDPK family. Besides, members within the same subfamily share a similar gene structure and motif composition. There are a large number of cis-elements involved in plant growth and response to stresses in the promoter regions of GbCDPKs. Additionally, these GbCDPKs show differential expression patterns in cotton tissues. The transcription levels of most genes were markedly altered in cotton under heat, cold, salt and PEG treatments, while the expressions of some GbCDPKs were induced in cotton under drought stress. Among these drought-induced genes, we selected GbCDPK32, GbCDPK68, GbCDPK74, GbCDPK80 and GbCDPK83 for further functional characterization by virus-induced gene silencing (VIGS) method. Conclusions: In conclusion, the principal findings of this prospective study are that CDPKs were associated with drought. These findings provide a solid foundation for the development of future molecular mechanism in sea-island cotton. 
1 Genome-wide identification and functional characterization of $2 C D P K$ gene family reveal their involvement in response to drought

3 stress in Gossypium barbadense

4 Guangzhen Shi ${ }^{1}$ and Xinxia Zhu ${ }^{1, *}$

$5{ }^{1}$ Key Laboratory of Oasis Town and Mountain-basin System Ecology of Xinjiang Production

6 and Construction Corps, College of Life Sciences, Shihezi University, Shihezi, Xinjiang, China;

7 Corresponding Author: Xinxia Zhu

8 College of Life Sciences, Shihezi University, Xinjiang, 832003, China

9 Email address: 302641316@qq.com

10 Abstract

11 Background: Calcium dependent protein kinases (CDPKs) are a class of important calcium signal sensing response proteins, which play an important regulatory role in response to abiotic stress. However, researchers have not been excavated CDPKs' role in drought in sea-island cotton(Gossypium barbadense L. 'H7124').

Results: $84 C D P K$ genes have been identified in G. barbadense. All of GbCDPK genes were disproportionately distributed on 26 chromosomes, and were categorized into four groups. Meanwhiles, segmental duplication was the predominant way for the extension of CDPK family. Besides, members of the same subfamily have similar gene structure and motif composition. There are many cis-elements in the promoter that are involved in plant growth and stress response. In addition, it revealed that all of GbCDPKs were differential expression patterns in cotton tissues. The transcriptional expression of most genes in cotton changed significantly under different treatments(heat, cold, $\mathrm{NaCl}$ and PEG), while the expression of some genes in cotton was induced under drought stress. Among these drought-inducing genes, we use the virusinduced gene silencing (VIGS) method to analyzed GbCDPK32, GbCDPK68, GbCDPK74, $G b C D P K 80$, and $G b C D P K 83$ for further function.

Conclusions: In conclusion, the principal findings of this prospective study are that CDPKs were associated with drought. These findings provide a solid foundation for the development of future molecular mechanism in sea-island cotton.

Keywords: Gossypium barbadense; Calcium dependent protein kinases (CDPKs); Virusinduced gene silencing (VIGS); Drought

Abbreviations: CDPKs, Calcium dependent protein kinases; VIGS, virus-induced gene silencing; qRT-PCR, quantitative reverse transcription PCR; G. barbadense, Gossypium barbadense; RWC, Relative water content; REL, Relative electrical conductivity leakage; MDA, Malondialdehyde.

Cotton is a significant financial and oil crop in the world, which provides the most commonly natural fiber materials for cotton textile industry. Hence, cotton has been developed for a huge scope around the world. Xinjiang is the largest cotton-growing region in China and even in the world. Sea-island cotton is the second-largest cotton planted in the world, which has shown in comparable superiority for its highly desirable fiber properties (Yu et al., 2021), which is grown in large areas in southern Xinjiang, where it is arid, with long sunlight and little rainfall. The growing area of sea-island cotton is limited due to the relatively low lint yield and narrow 
42 adaptation. Drought is one of the most serious climatic disasters affecting cotton quality and

43 production (Ault et al., 2020). This is a problem that needs to be solved urgently.

44 Calcium dependent protein kinases are a class of Ser/Thr type protein kinases, which are

45 usually composed of four core domains: N-terminal variable domain, protein kinase domain,

46 autoinhibitory domain and C-terminal calmodulin like domain (Cheng et al., 2002). Plants sense

47 environmental stimuli and transmit extracellular signals into intracellular through typical

48 mechanisms and cause a series of responses. The C-terminal calmodulin like domain is

49 composed of a pair of helix loop EF-hands structure. The combination of $\mathrm{Ca}^{2+}$ and EF-hands

50 structure can cause the change of the spatial structure of CDPKs, which leads to the release of

51 the inhibition of the self inhibitory domain, and promotes the recovery of the protein kinase

52 activity of CDPKs, phosphorylates the downstream regulatory factors, and transmits the signal to

53 the downstream regulatory network (Shi et al., 2018).

When plants are faced with abiotic stresses, CDPKs can respond to abiotic stresses and improve plant resistance to abiotic stresses by regulating the specific expression of genes. Overexpression of $A t C P K 4, A t C P K 8, A t C P K 10$ and $A t C P K 11$ can significantly improve the drought resistance of transgenic plants (Zou et al. 2010; Zhu et al. 2007; Zou et al. 2015). It is reported that $O s C D P K 4$ plays an important role in salt tolerance and drought stress of rice (Campo et al., 2014). Overexpression of OsCDPK9 play an active role in drought stress tolerance (Wei et al., 2014). Overexpression of tobacco ZoCDPK1 gene can affect salt and drought stress (Vivek et al., 2017). Overexpression of $Z m C P K 4$ in the transgenic Arabidopsis enhanced drought stress tolerance (Jiang et al., 2013).

Although CDPK genome-wide identification and functional studies have been carried out in Arabidopsis (34) (Hrabak et al., 2003), rice (31) (Ray et al., 2007) and maize (31) (Kong et al.,2013), there is no report on $C D P K$ gene family in G. barbadense. Based on G. barbadense genomes, we used bioinformatics to identify the members of $C D P K$ gene family and predict the molecular structure, physiological characteristics of the protein. Analyzing the chromosome location, evolution and classification, the expression profiles of the $C D P K$ gene. Moreover, we further investigated the function of the selected CDPKs in G. barbadense defense against drought stress by using virus-induced gene silencing (VIGS) technology. The results of this experiment provide an important theoretical basis for further understanding of the evolution and function of the $C D P K$ and the cultivation of drought-tolerant cotton varieties in G. barbadense.

74

75

76

77

78

79

80

81

\section{Materials \& Methods}

\section{Identification of the CDPK family genes in Gossypium barbadense}

The G. barbadense genome data comes from https://cottonfgd.org/ (Zhu et al., 2017), The Arabidopsis CDPK protein sequences of family members were downloaded from the Arabidopsis genome database TAIR website(https://www.arabidopsis.org/). Using Pfam online database(http://pfam.xfam.org/) to download the CDPK gene seed file EF-hand (Pfam ID: PF00036) and serine/threonine protein kinase (Pfam ID: PF07714). The hmmsearch program of HMMER3.0 was used to identify protein sequences containing CDPKs conserved domains. All CDPK protein sequences were extracted NCBI CDD and Pfam online database were used to 
82 verify the conservative domain. Finally, all members of $C D P K$ gene family were obtained and

83

84

85

86

87

88

89

90

91

92

93

94

95

96

97

98

99

100

101

102

103

104

105

106

107

108

109

110

111

112

113

114

115

116

117

118

119

120

121

named according to their different positions on chromosomes in G. barbadense.

\section{Molecular structure and physicochemical properties}

The Protparamtool (http://web.ExPasy.orP/ProtParam/)was used to predict the molecular weight, isoelectric point and other relevant information of the CDPK protein. Using the online tool myristoylation (https://web.expasy.org/myristoylator/) predict $N$-myristoylation, and palmitoylation prediction tools is CSS-plam. Subcellular localization of the CDPK proteins were predicted using an online tool wolf POSRT (https://www.genscript.com/psort.html).

\section{Phylogenetic tree, gene structure, conserved motif, and promoter region analyses}

The ClustalX software was used to perform multiple sequence alignments on the CDPK proteins of G. barbadense and Arabidopsis, and the phylogenetic tree was constructed by MEGAX software: the Neighbor-Joining algorithm was used, and the Bootstrap repeat value was 1000 times (Kumar et al., 2018). The online GSDS tool (http://gsds.cbi.pku.edu.cn) (Hu et al.,2015) was used t-o visualize the exon-intron structures of the CDPK genes. Using MEME (http://memesuite.org/tools/meme) (Bailey et al., 2009) online software to analyze the conserved motifs of $G$. barbaden-se CDPK, the parameters were set as the maximum number of motifs found was 10 , and the longest motif length was $100 \mathrm{nt}$ (Nucleotide). The $1500 \mathrm{bp}$ DNA sequence upstream was extracted, and the PlantCARE (Lescot et al., 2002) database

(http://bioinformatics.psb.ugent.be/webtools/plantcare/html/) was used to predict the possible cis-acting elements.

\section{Chromosome location and gene replication}

The information of $C D P K$ gene family chromosome physical location was extracted from $G$. barbadense genome annotation file, and the chromosome physical distribution map of the genes was drawn with MapInspect. The definition of gene duplication is as follows: (1) the sequence length is greater than $80 \%$ of the gene; (2) the identity of alignment region is greater than $80 \%$; (3) only one repeat event is calculated for closely linked genes. According to the chromosomal location of $C D P K$ gene, tandem duplication and segment duplication can be identified. The KaKs_Calculator2.0 of Tbtools (Chen et al., 2020) was used to calculate the non-synonymous mutation rate $(\mathrm{Ka})$ and the synonymous mutation rate $(\mathrm{Ks})$ of the replication gene pair in $G$. barbadense.

\section{Expression profile analysis, RNA extraction and real-time PCR}

The transcriptome data of $G b C D P K$ genes in the tissues and the abiotic stresses were from Sequence read archive, SRA, and the accession number was PRJNA274882. The RPKM value (the reads per kilobase of transcript per million mapped reads) represents the abundance of gene expression at the transcription level. Using Tbtools (Chen et al., 2020) map the gene expression profiles of different tissues and different abiotic stresses. Total RNA is extracted with the plant total RNA extraction kit (TIANGEN, Beijing, China). RNA reverse transcription adopts HiScript II QRT SuperMix for qPCR (R222-01), a product of Nanjing Novazin. The cDNA was used as a template to perform RT-PCR and qPCR experiments. The internal reference gene is the ubiquitinated protein Ubiquitin7 (UBQ7), and the RT-PCR reaction uses 1.0\% agarose gel to 
122 detect the amplification results. The quantitative instrument uses Roche products, and the 123 calculated $\mathrm{Ct}$ value is used to calculate the relative expression of the gene. The calculation 124 method is $2^{-\Delta \Delta \mathrm{Ct}}$ (Pfaffl et al.,2001). Each gene amplification result contains at least 3 technical 125 replicates and 3 biological replicates. The amplification system and procedure are shown in 126 Appendix A and Appendix B.

\section{Experiment treatment}

128 The G. barbadense was grown for 4 weeks in a high humidity and high nutrient environment 129 (nu-tritional soil: vermiculite: perlite $=3: 1: 1$ ) at $28^{\circ} \mathrm{C}$, and then treated with abiotic stress to well 130 gro--wing cotton seedlings. Cold stress and high temperature stress are moved plants to 131 incubators at $4^{\circ} \mathrm{C}$ and $37^{\circ} \mathrm{C}$, respectively. For salt stress, seedlings are irrigated with $200 \mathrm{mM}$ $132 \mathrm{NaCl}$. Seedlings are irrigated with 20\% PEG6000 for drought stress. One hour after the start of

133 the treatment, the second true leaf of the treated cotton seedling was harvested and stored in a 134 refrigerator at $-80^{\circ} \mathrm{C}$. Each treatment included 3 seedlings, and each treatment had three 135 experimental replicates.

\section{VIGS experiment in G. barbadense}

137 The silenced fragments of each $C D P K$ gene were amplified from cotton and inserted into VIGS 138 vector (TRV-00) to generate $C D P K$ silent constructs. TRV-00 without the silent segment was 139 used as control. Consequently, the vector was transformed into the GV3101. GV3101 contained 140 the same amount of TRV-00 and TRV-CDPK, and infiltrated into the cotyledons of 14-days-old 141 cotton seedlings by syringe infiltration method. Two weeks after inoculation, the silenced cotton 142 seedlings were used for gene expression analysis and identification PEG treatment.

\section{Methods of physiological index}

144 RWC was measured by using the formula: $\mathrm{RWC}(\%)=\frac{F W-D W}{T W-D W} \times 100$. Where FW, stands for 145 fresh weight TW for turgid weight and DW for dry weight (Loutfy et al., 2012). G. barbadense 146 leaves were soaked in $30 \mathrm{~mL}$ deionized water at $25^{\circ} \mathrm{C}$ and then shaken for 30 minutes. Heated the 147 solution to $100^{\circ} \mathrm{C}$ for 30 minutes and then at room temperature. Measure REL with conductivity 148 meter (Yang et al., 2012). The content of MDA, Pro, soluble sugar measured using the fresh 149 sample. The amount of MDA was measured by the thiobarbituric acid method (Heath \& Packer, 150 1968). The content of Pro was determined by the Sulfosalicylic acid indandione method (Bates 151 et al., 1973). Soluble sugar content was measured as described by the anthrone method and determined at $620 \mathrm{~nm}$ by a spectrophotometer(Fales, 1951). Water 1 oss $\operatorname{rate}(\%)=\frac{W 1-W 2}{W 1}$ $\times 100$.W1 stands for weight of detached blade before water loss.W2 weight of detached blade after 1, 2,3,4 and 5 hours of water loss(Czyczyło-Mysza et al.,2018). All physiological results were repeated three times and a one-way analysis of variance (ANOVA) was performed with Prism to detect significant differences between both treatments $(P<0.05, P<0.01)$. 
161

162

163

164

165

166

167

168

169

170

171

172

173

174

175

176

177

178

179

180

181

182

183

184

185

186

187

188

189

190

191

192

193

194

195

196

197

198

199

200

GbCDPK gene family members. After NCBI-CDD and Pfam software database verification, 84 $C D P K$ genes were identified in G. barbadense genome (Supplementary Table S1).

The biochemical characteristics of the $C D P K$ gene, such as amino acid sequence, molecular weight and isoelectric point were analyzed by the ProtParamtool online software. The results show that the CDPK genes are conservative. The longest CDPK protein encodes 648 amino acids, and the shortest only encodes 155 amino acids. The molecular weight of GbCDPKs varies $17.99 \mathrm{KDa}$ to $71.854 \mathrm{KDa}$, and the theoretical isoelectric point ranges from 4.313 to 9.481 . Multiple sequence alignment analysis indicated that all of GbCDPKs have four core domains. In addition, all the GbCDPKs have EF-hands motifs, which allows binding of $\mathrm{Ca}^{2+}$. It reported that 71 GbCDPK proteins contain 4 EF-hands, 9 proteins (GbCDPK25, GbCDPK26, GbCDPK39, GbCDPK49, GbCDPK53, GbCDPK67, GbCDPK68, GbCDPK77, GbCDPK81) have 3 EFhands, and remaining 4 proteins (GbCDPK22, GbCDPK38, GbCDPK61, GbCDPK64) only contains 2 EF-hands. Our bioinformatic analysis revealed that 40 members harbored potential $\mathrm{N}$ myristoylation sites, and 74 GbCDPK proteins contained S-palmitoylation sites (Supplementary Table S1). Using TMHMMServerv2.0 prediction, it was found that none of the 84 CDPK proteins had a transmembrane structure. Using Wolf POSRT tool to predict subcellular localization, it was found that 22 CDPK proteins are in the chloroplast, 33 CDPK proteins are in the cytoplasm, 8 are in the nucleus, and the others are in the mitochondria, extracellular, vacuole, endoplasmic reticulum, and peroxisome.

\section{Phylogenetic analysis of GbCDPKs}

To assess the phylogenetic relationship of CDPK protein in G. barbadense. A total of 118 CDPK proteins from G. barbadense and Arabidopsis were used to construct the phylogenetic tree by adjacency method (Figure 1). According to the results of phylogenetic tree clustering, $84 C D P K$ genes can be divided into four subfamilies. Referring to the research results of Arabidopsis, four subfamilies were named as class I, class II, class III, and class IV. Further analysis of the clustering results of CDPK members revealed that the gene structures in the homologous gene pair were similar. The number of subgroup II proteins was the largest, including $30 G$. barbadense and 10 Arabidopsis CDPK proteins, accounting for $35.71 \%$ and $29.41 \%$ of the total, respectively. The first subgroup includes $27 \mathrm{CDPK}$ proteins from G. barbadense and $10 \mathrm{CDPK}$ proteins from Arabidopsis. The third subgroup includes 19 CDPK proteins from G. barbadense and $8 \mathrm{CDPK}$ proteins from Arabidopsis. The fourth subgroup has the least number, including only 8 CDPK proteins from G. barbadense and 3 CDPK proteins from Arabidopsis, indicating that it's far from each other.

\section{Chromosomal distribution and gene duplication}

To investigate the chromosomal locations, chromosomal maps were drawn for the $84 C D P K$ genes from $G$. barbadense by MapInspect software. All 84 GbCDPK genes are mapped on 13 pairs of chromosomes (Figure 2). Furthermore, cotton genome includes A-subgroup and Dsubgroup that all contain $42 \mathrm{GbCDPKs}$, respectively, and the duplication events may illuminate the mechanism about the expansion of $G b C D P K$ gene family. There are 6 genes on chromosome 4 and 13 of A-subgroup, 5 genes on chromosome 2 of A-subgroup and 5 and 13 of D-subgroup, 
201 respectively. In contrast, there is only one gene on chromosome 3 and 8 of A-subgroup and 202 chromosome 8 of D-subgroup, and there are 2-4 genes on other chromosomes. These results 203 indicated that $C D P K$ genes were widely distributed in G. barbadense. In order to speculate the 204 possible relationship between $G b C D P K$ genes and potential gene replication in the $G$. 205 barbadense genomes, we analyzed the occurrence of tandem duplication and large-scale 206 segmental replication during the evolution of the gene family (supplementary Table S2). Among 20784 genes, 41 pairs of homologous genes were found in phylogenetic tree (Figure 1). There were 20840 pairs of segmental replications and one pair of tandem replications (GbCDPK10/GbCDPK11). 209 To study the evolutionary replication relationship of genes in G. barbadense on chromosome 210 segments, MCscanx software was used to detect the replication genes. 12, 16 and 60 pairs of 211 whole genome replicates were detected among $\mathrm{A}, \mathrm{D}$ and $\mathrm{AD}$ subgroups in G. barbadense, 212 respectively (Figure 2). KaKs_Calculator was used to perform $\mathrm{Ka} / \mathrm{Ks}$ analysis replication gene 213 pairs. It was found that the $\mathrm{Ka} / \mathrm{Ks}$ of all gene pairs were less than 1 , indicating that the $C D P K$ 214 gene of $G$. barbadense might have undergone strict purification and selection during the 215 evolution process, implying that the replication gene is evolutionarily conserved, structurally 216 stable, and might have consistent function.

217 Analysis of gene structure and conserved motifs

218 The $C D P K$ genes were visualized for the gene structure and conserved motifs. The results 219 showed that they have similar gene structure distribution patterns in the same subfamily. Most 220 CDPKs of the first and the third subfamily have 7 or 8 exons, the CDPKs of the second 221 subfamily have 8 or 9 exons, and the fourth subfamily has 12 exons. Most CDPKs of the family 222 have 12 exons (Figure 3). Generally, the structural conservation and similarity of the same type 223 of genes are closely related to their evolutionary relationship, but there are some exceptions. $224 G b C D P K 38$ has only 5 exons and GbCDPK61 has only 3 exons. The longest exon of most $225 \mathrm{GbCDPKs}$ is the first exon. However, there are still a small number of genes in the family 226 members that have evolved significantly different structures. GbCDPK39 and GbCDPK81 are 227 homologous genes from different sub-genomes, GbCDPK39 has a typical gene structure of this 228 group, while the first exon of GbCDPK81 is relatively short. GbCDPK10 and GbCDPK11 are 229 homologous genes of the same sub-genomes, but their gene structure is also different. $230 G b C D P K 10$ has typical gene structure characteristics, but the first exon of GbCDPK11 is also 231 shorter than that of GbCDPK10. This structural difference is likely to have formed randomly 232 during evolution. The changed gene structure may lead to changes in gene splicing sites, thereby 233 generating new transcripts or non-functional gene products, and ultimately increasing the number 234 of members of the cotton GbCDPKs gene family.

235 The MEME online software was used to conduct conservative analysis on the amino acid 236 sequence of CDPK, and 10 conservative motifs were found (Figure 4). These 10 conserved 237 motifs exist in subgroups I, II, III and IV, but there are four genes (GbCDPK11, GbCDPK14, $238 G b C D P K 38, G b C D P K 61)$ whose conservative motifs are distributed differently in the first 239 subgroup. GbCDPK11, GbCDPK14 contains the same conserved motifs, without Motif 2, Motif 240 3, Motif 5. While GbCDPK38 has only 3 conserved motifs (Motif 8, Motif 9, Motif 10), 
$241 G b C D P K 61$ has 2 conserved motifs (Motif 8, Motif 10). However, in the same subfamily, $C D P K$ 242 has a similar number, type, and spatial distribution of motifs, demonstrating that the functions of $243 C D P K$ genes in the same subfamily are similar.

\section{Analysis of promoter cis-elements}

245 To explore the regulatory mechanisms of $G b C D P K$ genes, the $1.5 \mathrm{~kb}$ sequence upstream of the 246 start condon of the GbCDPKs was selected to analyze the constitutions of cis-regulatory 247 elements. It was found that all CDPK genes contained CAAT-box conserved elements and 248 TATA-box. In addition to these conserved elements, there are four types of cis-regulatory elements in the promoter of $C D P K$ gene: (1) Light regulatory elements, including Box 4, G-Box,

250

251

252

253

254

255

256

257

258

259

260

261

262

263

264

265

266

267

268

269

270

271

272

273

274

275

276

277

278

279

280

TCCC-motif and AE-box, etc.(2) Plant growth and development regulatory elements, including CAT-box, GCN4_motif, O2-site, MBSI and circadian, etc.(3) Phytohormone response elements, including methyljasmonate response elements TGACG-motif and CGTCA-motif, (ABRE), gibberellin responsive element P-box, etc.(4) Stress response elements include LTR, MBS, ARE and mechanical damage response element W-box, etc.

\section{Expression profile of different tissues and abiotic stress responses}

To explore the possible biological functions of GbCDPKs, the transcriptome data of $G$. barbadense were used to analyze the expression characteristics of gene family in ovule and fiber at $0,1,3,5,10$ and 20 days after anthesis, as well as in root, stem, leaf, receptacle, petal, stamen, calycle and sepals (Figure 5A). The results showed that the CDPK gene has tissue-specific expression and spatio-temporal expression characteristics. According to the expression characteristics, the CDPK family can be divided into three types: the first group of genes are generally expressed less, but some genes were expressed in some or specific tissue. The second group was highly expressed in most tissues and developmental stages. The third group of genes are mainly expressed in vegetative organs or floral organs, but less expressed in ovule and fiber. Generally, homologous genes have similar expression patterns. Some homologous genes, such as $G b C D P K 13, G b C D P K 56$ and $G b C D P K 30, G b C D P K 72$ are highly expressed in the stamens. GbCDPK28, GbCDPK70; GbCDPK33, GbCDPK75 and GbCDPK38, GbCDPK80 are predominantly expressed in ovules. GbCDPK1, GbCDPK43 and GbCDPK25, GbCDPK67 are predominantly expressed in receptacles and stamens. The above results indicate that the $C D P K$ gene family is widely involved in the growth and development of G. barbadense.

CDPKs can regulate the response of plants to abiotic stress. The transcriptome data was used to analyze the expression characteristics of the $C D P K$ gene of $G$. barbadense under low temperature, high temperature, drought (PEG6000), and salt treatment (Figure 5B). According to the expression characteristics shown in response to abiotic stress, the CDPK gene family can be divided into 4 groups: the first group most genes are low expressed under adversity stress. The second group all genes is low expressed. The three groups are all highly expressed. The fourth group most genes are highly expressed. In addition, homologous genes have similar expression profiles. For example, some homologous genes GbCDPK31 and GbCDPK73 are highly expressed under all stresses.

\section{Expression under drought stress}

Peer) reviewing PDF | (2021:10:67134:2:1:NEW 9 Jan 2022) 
281 The transcript levels of $C D P K$ genes in cluster 1 and 2 were decreased under drought. In order to 282 further understand the expression of $C D P K$ genes at transcriptional level in G. barbadense. qRT283 PCR was performed using RNA isolated from drought stress (Figure 6). After drought, the 284 expression of $G b C D P K 4$ showed a downward-increasing-decreasing trend, and the expression 285 was the lowest at $6 \mathrm{~h}$. GbCDPK19 and GbCDPK78 showed an overall upward-decreasing286 increasing-decreasing trend. The former had the highest expression at $12 \mathrm{~h}$, and the latter at $12 \mathrm{~h}$. 287 The expression level of GbCDPK23 was the highest at $3 \mathrm{~h}$. The expression level of GbCDPK23 288 showed a trend of decrease-increasing-decreasing, and the expression level of $12 \mathrm{~h}$ was the 289 highest. The expression levels of GbCDPK44 and GbCDPK66 showed a trend of increase290 decrease-increasing, and the expression level of the former was the lowest at $6 \mathrm{~h}$. The expression 291 was highest at $24 \mathrm{~h}$.

\section{Silencing GbCDPKs compromises cotton resistance to drought stress}

293

294

295

296

297

298

299

300

301

302

303

304

305

306

307

308

309

310

311

312

313

314

315

316

317

318

319

320

In order to further study the function of CDPKs drought tolerance in G. barbadense, we chose to design specific primers in the 3'-UTR region of CDPKs to construct VIGS vectors. The transcript levels of $C D P K$ genes in cluster 3 and 4 were significantly increased under drought, with the highest peak observed all stage of the drought treatment. The empty vector (TRV-00) and the vector with target fragments (TRV-CDPKs) were activated and propagated. Then the cotton cotyledons were injected into the 10 days seedlings for gene silencing. After two weeks of injection, when the positive seedlings were albino (Figure 7A), indicating success of the VIGS experiment. The observed gene expression patterns may reflect their functions. The interference efficiency of the target gene was detected by qPCR technology. The results show that the GbCDPKs genes (CDPK32, CDPK68,CDPK74, CDPK80 and CDPK83) in cotton plants injected with bacterial liquid containing the target gene fragments can indeed interfere effectively and specifically (Figure 7B).

To determine the tolerance of GbCDPKs silenced plants under drought stress, leaf discs were made from the same parts of TRV-00 and TRV-GbCDPKs (TRV-CDPK32, TRV-CDPK68, TRV-CDPK74, TRV-CDPK80 and TRV-CDPK83). The leaves were incubated in 0 and $20 \%$ PEG6000 solution for 4 days. The results showed that the leaf chlorosis and wilting of gene silenced TRV-CDPK32, TRV-CDPK68, TRV-CDPK74, TRV-CDPK80 and TRV-CDPK83 plants were significantly more chlorosis and wilting than that of the control (Figure 7C).

In addition, we measured several physiological indicators including RWC, REL, water loss rate and MDA, proline and soluble sugar content under drought treatment and normal growth conditions. As shown in Figure7D-H, in normal conditions, relative water content, electrical conductivity, proline and soluble sugar content of TRV-00 were higher than TRV-GbCDPKs. However, the TRV-00 of MDA was lower than silencing. Under the drought stress, the relative water content, proline and soluble sugar content were decreased in TRV-CDPKs plants, compared with the TRV-00. The relative electrical conductivity, MDA of TRV-00 and TRVCDPKs both increased. From Figure 7I, TRV-CDPKs was significantly higher than TRV-00, indicating that the drought resistance of plants decreased remarkably after silenced. In conclusion, the drought tolerance of the plants is weaker after silenced.

PeerJ reviewing PDF | (2021:10:67134:2:1:NEW 9 Jan 2022) 


\section{Discussion}

322 In this study, we have identified 84 presumptive CDPK genes in G. barbadense (AADD). The

323

324

325

326

327

328

329

330

331

332

333

334

335

336

337

338

339

340

341

342

343

344

345

346

347

348

349

350

351

352

353

354

355

356

357

358

359

360 members of CDPKs family are significantly more than those in other species. There are 41 pairs of homologous gene pairs in the CDPK gene family, and 40 pairs are segmental duplication between the A/D chromosome pairs. Our analysis showed that segmental duplication is a predominant driving force that contributed to the expansion of GbCDPKs. The parallel evolution characteristics of homologous gene pairs in A-subgroup and D-subgroup are significant. They may come from the same ancestor, but the remaining one pair exists in the form of tandem replication(Liu et al.,2014). Generally, the amplification of CDPKs family members in angiosperms mainly depends on large-scale gene rearrangement, that is, whole genome or large fragment gene replication events (Hamel et al.,2013). This may explain the fact that there are more members of CDPKs family in G. barbadense than in Arabidopsis, rice and maize. Phylogenetic analysis indicated the GbCDPKs divided into four groups like Arabidopsis, and most CDPKs in the same group have similar gene structure, indicating the evolutionary and functional conservation of CDPKs. Generally, the orthologous genes have similar biological functions, while the paralogous genes have different biological functions. Therefore, the function of $G b C D P K$ genes can be inferred from orthologous genes to provide scientific theoretical basis for subsequent functional studies (Thornton et al., 2000).

The specificity of signal transduction can be determined by the subcellular location of CDPK protein. It is predicted that GbCDPKs are in several cell compartments. It is also predicted that most GbCDPKs contain $N$-myristoylation and palmitoylation. The CDPK genes of $N$ myristoylation motif tends to be in the plasma membrane (Martín et al., 2000; Lu et al., 2013) The myristoylation may be a part of the main signal transduction process that guides proteins to membrane binding sites. In addition, it is reported that another lipid-modified palmitoylation is necessary for the stability of membrane association (Asai et al., 2013). In this study, 40 genes have $N$-myristoylation and 75 genes have palmitoylation motifs. However, the association of CDPKs with membranes is complex and may be affected by other motifs. For example, in wheat (Triticum aestivum), TaCPK3 and TaCPK15 without myristoylation regions are associated with the membrane (Li et al., 2008), while in Maize (Zea mays), $N$-myristoylation patterns were found in $Z m C P K 1$ of the cytoplasm and nucleus (Wang et al.,2013).

Other studies have shown that the subcellular location of CDPKs can be limited in a single compartment or widely distributed in the whole cell. CDPKs have been found in plasma membrane, cytoplasm, nucleus, endoplasmic reticulum, mitochondria, chloroplast, oleosome, peroxisome and Golgi apparatus (Asano et al., 2012a). To explore the function of proteins in plant, subcellular localization is one of the essential research. $\mathrm{Ca}^{2+}$ pumps are known to be widely distributed on membranes(Tanaka et al., 2004), CDPK can rapidly respond to transient changes of $\mathrm{Ca}^{2+}$ signal in plants and specifically recognize substrates after phosphorylation. Various physiological responses were further triggered in signaling transduction cascades, which finally regulate plant growth and development, and in response to multiple stresses(Shi et al.,2018). We found that StCDPK7 protein with auto-phosphorylation activity could locate in the

Peer) reviewing PDF | (2021:10:67134:2:1:NEW 9 Jan 2022) 
361

362

363

364

365

366

367

368

369

370

371

372

373

374

375

376

377

378

379

380

381

382

383

384

385

386

387

388

389

390

391

392

393

394

395

396

397

398

399

400

nucleus, membranes and interact with PAL protein, suggesting that the auto-phosphorylation activity of StCDPK 7 protein played important roles in protein location and protein-protein interactions (Fantino et al., 2017). SiCDPK24 protein was proven to have a role in autophosphorylation, and may be important for its function. SiCDPK24-GFP localized to the plasma membrane and the nucleus(Yu et al., 2018). Therefore, the subcellular localization of CDPK genes are closely related to drought stress response.

CDPKs activated by transient changes in $\mathrm{Ca}^{2+}$ concentration in plants is essential for various biological processes (such as plant growth, development, regeneration, defense against biotic and abiotic stresses, etc.) (Asano et al., 2012a; Ranty et al., 2016). It has been reported that CDPKs are widely distributed in plant tissues, even in fruit, pollen tubes, germs, and guard cells (Chang et al., 1995; Zhou et al., 2009). The CDPKs protein family is huge, and its functions are also diverse (Asano et al., 2012b). We use the transcriptome data to evaluate the expression pattern of cotton CDPKs in 17 different tissues. The results of tissue expression distribution indicated that the gene family of cotton CDPKs was widely distributed, implying that its function is diverse. CDPKs expressed in many tissues may participate in a variety of biological processes, some members specifically expressed in stamens may participate in reproductive development, and some may participate in stress response process. The specific genes involved in which biological process need to be further studied.

With the change of global ecological environment, drought has become one of the main environmental factors limiting crop quality and yield. In other plants, CDPKs was associated with drought tolerance, such as StCDPK3 and StCDPK23 and PtrCDPK10 are all involved in drought stress (Bi et al., 2021; Meng et al., 2020). However, few people have studied the function of CDPKs in G. barbadense.

To further study the function of drought tolerance in GbCDPKs, VIGS technology was used to silence candidate $C D P K$ genes to construct transient silence materials (Senthil-Kumar et al., 2014). Our analysis indicated that CDPKs silencing plants were more sensitive to drought stress than control plants. The results of the study showed that the drought resistance ability of CDPKs silencing was significantly reduced. The content of proline and the MDA was higher than the control. On the contrary, the relative water content of the silenced plants was lower than that in the controls under drought stress. To maintain the stability of protoplast colloid, the proline is accumulated to increase the abiotic pressure of plant cells under drought stress (Sallam et al., 2019). In this study, drought stress induced accumulation of proline in cotton, and thereby enhanced abiotic stress adjustment ability. The drought sensitive phenotype of silencing plants to drought stress indicates that CDPKs play a regulatory role in improving cotton drought tolerance, but whether they participate in the same drought tolerance regulatory mechanism is still unknown, and further research is needed to prove it.

\section{Conclusions}

$84 C D P K$ genes were identified in G. barbadense. They all have the EF-hands structure and $\mathrm{Ser} / \mathrm{Thr}$ protein kinase domain, none of which include the transmembrane structure. They were found to express in different organelles by subcellular localization. According to the 
401 phylogenetic tree, all CDPKs were divided into four groups, which were distributed on 13 pairs

402 of chromosomes. The CDPKs are distributed in 13 tissues. In response to abiotic stress, it

403 showed different expression patterns. The silence of some CDPKs severely destroyed the basic

404 resistance of cotton to drought stress, indicating that they were involved in the resistance

405 mechanism. This study deepens our understanding of the function of CDPK and provide

406 important insights for further research on the molecular mechanism of G. barbadense to drought

407 stress and to provide candidate target genes that improve drought adaptation.

\section{Acknowledgements}

409 We would like to express gratitude to Prof. Zhang Tianzhen and Assoc. Prof. Fang Lei from

410 Zhejiang University for providing G. barbadense transcriptome data.

\section{References}

412 Asai S, Ichikawa T, Nomura H, Kobayashi M, Kamiyoshihara Y, Mori H, Kadota Y, Zipfel C, Jones JDG, Yoshioka H. 2013. The variable domain of a plant calcium-dependent protein kinase (CDPK) confers subcellular localization and substrate recognition for NADPH oxidase. J. Biol. Chem. 288(20):14332-14340. DOI: 10.1074/jbc.M112.448910.

Asano T, Hayashi N, Kikuchi S, Ohsugi R. 2012a. CDPK-mediated abiotic stress signaling. Plant Signal Behav. 7(7):817-21. DOI: 10.4161/psb.20351.

Asano T, Hayashi N, Kobayashi M, Aoki N, Miyao A, Mitsuhara I, Ichikawa H, Komatsu S, Hirochika H, Kikuchi S, Ohsugi R. 2012b. A rice calcium-dependent protein kinase OsCPK12 oppositely modulates salt-stress tolerance and blast disease resistance. Plant J. 69(1):26-36. DOI: 10.1111/j.1365-313X.2011.04766.x.

Ault TR. 2020. On the essentials of drought in a changing climate. Science 368(6488):256-260. DOI: $10.1126 /$ science.aaz5492.

Bates LS, Waldren RP, Teare ID. 1973. Rapid determination of free proline for water-stress studies. Plant and Soil 39(1):205-207. DOI:10.1007/BF00018060.

Bailey TL, Boden M, Buske FA, Frith M, Grant CE, Clementi L, Ren J, Li WW, Noble WS. 2009. MEME SUITE: tools for motif discovery and searching. Nucleic Acids Res. 37(Web Server issue): W202-8. DOI: 10.1093/nar/gkp335.

Bi Z, Wang Y, Li P, Sun C, Qin T, Bai J. 2021. Evolution and expression analysis of CDPK genes under drought stress in two varieties of potato. Biotechnol. Lett. 43(2):511-521. DOI: 10.1007/s10529-020-03037-2.

Bradford MM. 1976. A rapid and sensitive method for the quantitation of microgram quantities of protein utilizing the principle of protein-dye binding. Analytical Biochemistry $72(1-$ 2):248-254. DOI: 10.1006/abio.1976.9999.

Campo S, Baldrich P, Messeguer J, Lalanne E, Coca M, San SB. 2014. Overexpression of a calcium-dependent protein kinase confers salt and drought tolerance in rice by preventing membrane lipid peroxidation. Plant Physiol. 165(2):688-704. DOI: 10.1104/pp.113.230268.

Chang A, Condron R, Neumann GM, Polya GM. 1995. Purification and characterization of a heat-stable wheat substrate for wheat embryo calcium-dependent protein kinase. Biochim. Biophys. Acta. 1244(2-3):317-24. DOI: 10.1016/0304-4165(95) 00039-e.

Cheng SH, Willmann MR, Chen HC, Sheen J. 2002. Calcium signaling through protein kinases. The Arabidopsis calcium-dependent protein kinase gene family. Plant Physiol. 129(2):46985. DOI: $10.1104 / \mathrm{pp} .005645$.

Chen C, Chen H, Zhang Y, Thomas HR, Frank MH, He Y, Xia R. 2020. TBtools: An integrative toolkit developed for interactive analyses of big biological data. Mol. Plant 13(8):11941202. DOI: 10.1016/j.molp.2020.06.009. 
447

448

449

450

451

452

453

454

455

456

457

458

459

460

461

462

463

464

465

466

467

468

469

470

471

472

473

474

475

476

477

478

479

480

481

482

483

484

485

486

487

488

489

490

Czyczyło-Mysza IM, Marcińska I, Skrzypek E, Bocianowski J, Dziurka K, Rančić D, Radošević R, Pekić-Quarrie S, Dodig D, Quarrie SA. 2018. Genetic analysis of water loss of excised le aves associated with drought tolerance in wheat. PeerJ 6:e5063. DOI: 10.7717/peerj.5063.

Fales FW. 1951. The assimilation and degradation of carbohydrates by yeast cells. Journal of Biological Chemistry 193:113-124. DOI: 10.1007/s10482-013-0100-5.

Fantino E, Segretin M E, Santin F, Mirkin F G, Ulloa RM. 2017. Analysis of the potato calciumdependent protein kinase family and characterization of stcdpk7, a member induced upon infection with Phytophthora infestans. Plant Cell Rep. 36 1137-1157. DOI:10.1007/s00299017-2144-x

Hamel LP, Sheen J, Séguin A. 2014. Ancient signals: comparative genomics of green plant CDPKs. Trends Plant Sci. 19(2):79-89. DOI: 10.1016/j.tplants.2013.10.009.

Heath RL, Packer L. 1968. Photoperoxidation in isolated chloroplasts. I-kinetics and stoichiometry of fatty acid peroxidation. Archives of Biochemistry and Biophysics 125(1):189-198. DOI: 10.1016/0003-9861(68)90654-1.

Hrabak EM, Chan CW, Gribskov M, Harper JF, Choi JH, Halford N, Kudla J, Luan S, Nimmo HG, Sussman MR, Thomas M, Walker-Simmons K, Zhu JK, Harmon AC. 2003. The Arabidopsis CDPK-SnRK superfamily of protein kinases. Plant Physiol. 132(2):666-80. DOI: $10.1104 / p p .102 .011999$.

Hu B, Jin J, Guo AY, Zhang H, Luo J, Gao G. 2015. GSDS 2.0: an upgraded gene features visualization server. Bioinformatics 31(8):1296-7. DOI: 10.1093/bioinformatics/btu817.

Jiang S, Zhang D, Wang L, Pan J, Liu Y, Kong X, Zhou Y, Li D. 2013. A maize calciumdependent protein kinase gene, $Z m C P K 4$, positively regulated abscisic acid signaling and enhanced drought stress tolerance in transgenic Arabidopsis. Plant Physiol. Biochem. 71:112-20. DOI: 10.1016/j.plaphy.

Kong X, Lv W, Jiang S, Zhang D, Cai G, Pan J, Li D. 2013. Genome-wide identification and expression analysis of calcium-dependent protein kinase in maize. BMC Genomics. 14:433. DOI: 10.1186/1471-2164-14-433.

Kumar S, Stecher G, Li M, Knyaz C, Tamura K. 2018. MEGA X: Molecular evolutionary genetics analysis across computing platforms. Mol. Biol. Evol. 35(6):1547-1549. DOI: 10.1093/molbev/msy096.

Lescot M, Déhais P, Thijs G, Marchal K, Moreau Y, Van Y, Rouzé P, Rombauts S. 2002. PlantCARE, a database of plant cis-acting regulatory elements and a portal to tools for in silico analysis of promoter sequences. Nucleic Acids Res. 30(1):325-7. DOI: 10.1093/nar/30.1.325.

Li AL, Zhu YF, Tan XM, Wang X, Wei B, Guo HZ, Zhang ZL, Chen XB, Zhao GY, Kong XY, Jia JZ, Mao L. 2008. Evolutionary and functional study of the CDPK gene family in wheat (Triticum aestivum L.). Plant Mol. Biol. 66(4):429-43. DOI: 10.1007/s11103-007-9281-5.

Liu W, Li W, He Q, Daud MK, Chen J, Zhu S. 2014. Genome-wide survey and expression analysis of calcium-dependent protein kinase in Gossypium raimondii. PLoS One 9(6):e98189. DOI: 10.1371/journal.pone.0098189.

Loutfy N, ElTayeb MA, Hassanen AM, Moustafa MFM, Sakuma Y, Inouhe M. 2012. Changes in the water status and osmotic solute contents in response to drought and salicylic acid treatments in four different cultivars of wheat (Triticum aestivum). Journal of Plant Research 12(1):173-184. DOI: 10.1007/s10265-011-0419-9. 
491

492

493

494

495

496

497

498

499

500

501

502

503

504

505

506

507

508

509

510

511

512

513

514

515

516

517

518

519

520

521

522

523

524

525

526

527

528

529

530

531

532

533

534

535

536

537

538

Lu SX, Hrabak EM. 2013. The myristoylated amino-terminus of an Arabidopsis calciumdependent protein kinase mediates plasma membrane localization. Plant Mol. Biol. 82(3):267-78. DOI: 10.1007/s11103-013-0061-0.

Martín ML, Busconi L. 2000. Membrane localization of a rice calcium-dependent protein kinase (CDPK) is mediated by myristoylation and palmitoylation. Plant J. 24(4):429-35. DOI: 10.1046/j.1365-313x.2000.00889.x.

Meng L, Zhang Q, Yang J, Xie G, Liu JH. 2020. PtrCDPK10 of poncirus trifoliata functions in dehydration and drought tolerance by reducing ROS accumulation via phosphorylating PtrAPX. Plant Sci. 291:110320. DOI: 10.1016/j.plantsci.2019.110320.

Pfaffl MW. 2001. A new mathematical model for relative quantification in real-time RT-PCR. Nucleic Acids Res. 29(9): e45. DOI: 10.1093/nar/29.9.e45.

Ray S, Agarwal P, Arora R, Kapoor S, Tyagi AK. 2007. Expression analysis of calciumdependent protein kinase gene family during reproductive development and abiotic stress conditions in rice (Oryza sativa L. Ssp. indica). Mol. Gen. Genomics. 278(5):493-505. 7. DOI: $10.1007 / \mathrm{s} 00438-007-0267-4$.

Ranty B, Aldon D, Cotelle V, Galaud JP, Thuleau P, Mazars C. 2016. Calcium sensors as key hubs in plant responses to biotic and abiotic stresses. Front Plant Sci. 7:327. DOI: 10.3389/fpls.2016.00327.

Sallam A, Alqudah AM, Dawood MFA, Baenziger PS, Börner A. 2019. Drought stress tolerance in wheat and barley: advances in physiology, breeding and genetics research. Int. J. Mol. Sci. 20(13):3137. DOI: 10.3390/ijms20133137.

Senthil-Kumar M, Mysore KS. 2014. Tobacco rattle virus-based virus-induced gene silencing in Nicotiana benthamiana. Nat. Protoc. 9(7):1549-62. DOI: 10.1038/nprot.2014.092.

Shi S, Li S, Asim M, Mao J, Xu D, Ullah Z, Liu G, Wang Q, Liu H. 2018. The Arabidopsis calcium-dependent protein kinases (CDPKs) and their roles in plant growth regulation and abiotic stress responses. Int. J. Mol. Sci. 19(7):1900. DOI: 10.3390/ijms19071900.

Tanaka M, Fujita H, Handa S, Murayama M, Uemura Y, Kawamura T, Mitsui S, Mikami Y, Tozawa T, Yoshinaga \& S. Komatsu. 2004. Proteomics of the ricecell:systematic identification of the protein populations in sub-cellular compartments. Mol Genet Genomics 271 (5) :566-576.

Thornton JW, DeSalle R. 2000. Gene family evolution and homology: genomics meets phylogen-etics. Annu. Rev. Genomics Hum. Genet. 1:41-73. DOI: 10.1146/annurev.genom.1.1.41.

Vivek PJ, Resmi MS, Sreekumar S, Sivakumar KC, Tuteja N, Soniya EV. 2017. Calciumdependent protein kinase in ginger binds with importin- $\alpha$ through its junction domain for nuclear localization, and further interacts with NAC transcription factor. Front Plant Sci. 7:1909. DOI: 10.3389/fpls.2016.01909.

Wang CT, Shao JM. 2013. Characterization of the ZmCPK1 gene encoding a Calcium-dependent protein kinase responsive to multiple abiotic stresses in maize. Plant Mol. Biol. Rep. 31, 222-230. DOI: 10.1007/s11105-012-0496-5.

Wei S, Hu W, Deng X, Zhang Y, Liu X, Zhao X, Luo Q, Jin Z, Li Y, Zhou S, Sun T, Wang L, Yang G, He G. 2014. A rice calcium-dependent protein kinase $O s C P K 9$ positively regulates drought stress tolerance and spikelet fertility. BMC Plant Biol. 14:133. DOI: 10.1186/14712229-14-133.

Yang Q, Rao J, Yi S, Meng K, Wu J, Hou Y. 2012. Antioxidant enzyme activity and chilling injury during low-temperature storage of Kiwifruit cv. Hongyang exposed to gradual postharvest cooling. Horticulture, Environment, and Biotechnology 53(6):505-512. DOI:10.1007/s13580-012-0101-8. 
539

540

541

542

543

544

545

546

547

548

549

550

551

552

553

554

555

556

557

558

559

560

561

562

563
Yu J, Hui Y, Chen J, Yu H, Gao X, Zhang Z, Li Q, Zhu S, Zhao T. 2021. Whole-genome resequencing of 240 Gossypium barbadense accessions reveals genetic variation and genes associated with fiber strength and lint percentage. Theor. Appl. Genet. 134(10):3249-3261. DOI: 10.1007/s00122-021-03889-w.

Yu TF, Zhao WY, Fu JD, Liu YW, Chen M, Zhou YB, Ma YZ, Xu ZS, Xi YJ. Genome-Wide Analysis of CDPK Family in Foxtail Millet and Determination of SiCDPK24 Functions in Drought Stress. Front Plant Sci. 2018 Jul 26;9:651. DOI: 10.3389/fpls.2018.00651.

Zou JJ, Wei FJ, Wang C, Wu JJ, Ratnasekera D, Liu WX, Wu WH. 2010. Arabidopsis calciumdependent protein kinase $C P K 10$ functions in abscisic acid and $\mathrm{Ca}^{2+}$-mediated stomatal regulation in response to drought stress. Plant Physiol. 154(3):1232-43. DOI: $10.1104 / \mathrm{pp} .110 .157545$.

Zou JJ, Li XD, Ratnasekera D, Wang C, Liu WX, Song LF, Zhang WZ, Wu WH (2015). Arabidopsis CALCIUM-DEPENDENT PROTEIN KINASE8 and CATALASE3 function in abscisic acid-mediated signaling and $\mathrm{H}_{2} \mathrm{O}_{2}$ homeostasis in stomatal guard cells under drought stress. Plant Cell 27(5):1445-60. DOI: 10.1105/tpc.15.00144.

Zhou L, Fu Y, Yang Z. 2009. A genome-wide functional characterization of Arabidopsis regulatory calcium sensors in pollen tubes. J. Integr. Plant Biol. 51(8):751-61. DOI: 10.1111/j.1744-7909.2009.00847.x.

Zhu T, Liang C, Meng Z, Sun G, Meng Z, Guo S, Zhang R. 2017. CottonFGD: an integrated functional genomics database for cotton. BMC Plant Biol. 17(1):101. DOI: 10.1186/s12870017-1039-X.

Zhu SY, Yu XC, Wang XJ, Zhao R, Li Y, Fan RC, Shang Y, Du SY, Wang XF, Wu FQ, Xu YH, Zhang XY, Zhang DP. 2007. Two calcium-dependent protein kinases, CPK4 and CPK11, regulate abscisic acid signal transduction in Arabidopsis. Plant Cell 19(10):3019-36. DOI: $10.1105 /$ tpc. 107.050666 . 
Figure 1

Evolutionary relationship of CDPK Family in G. barbadense 


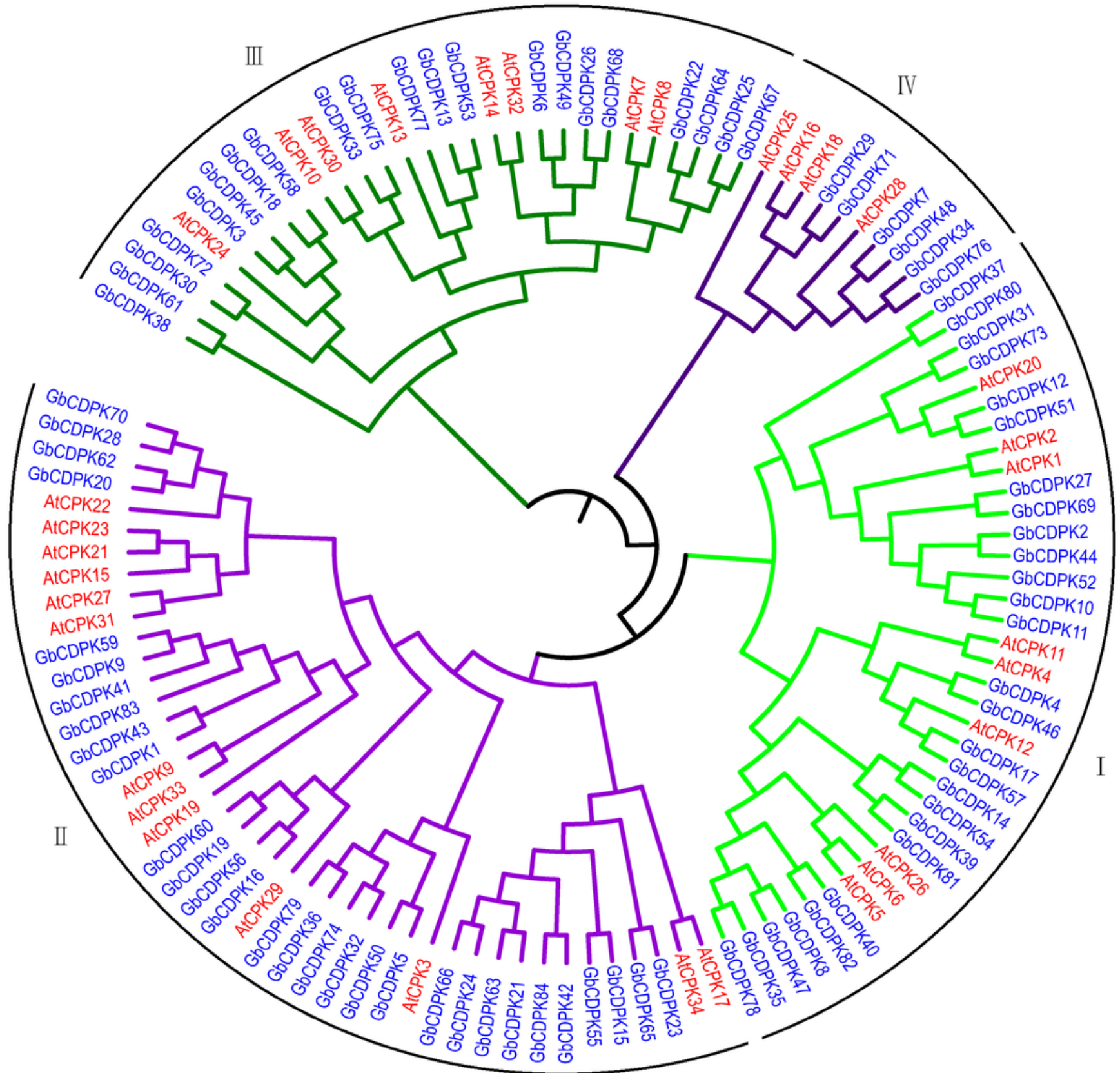


Figure 2

Distribution of CDPK gene family on chromosomes

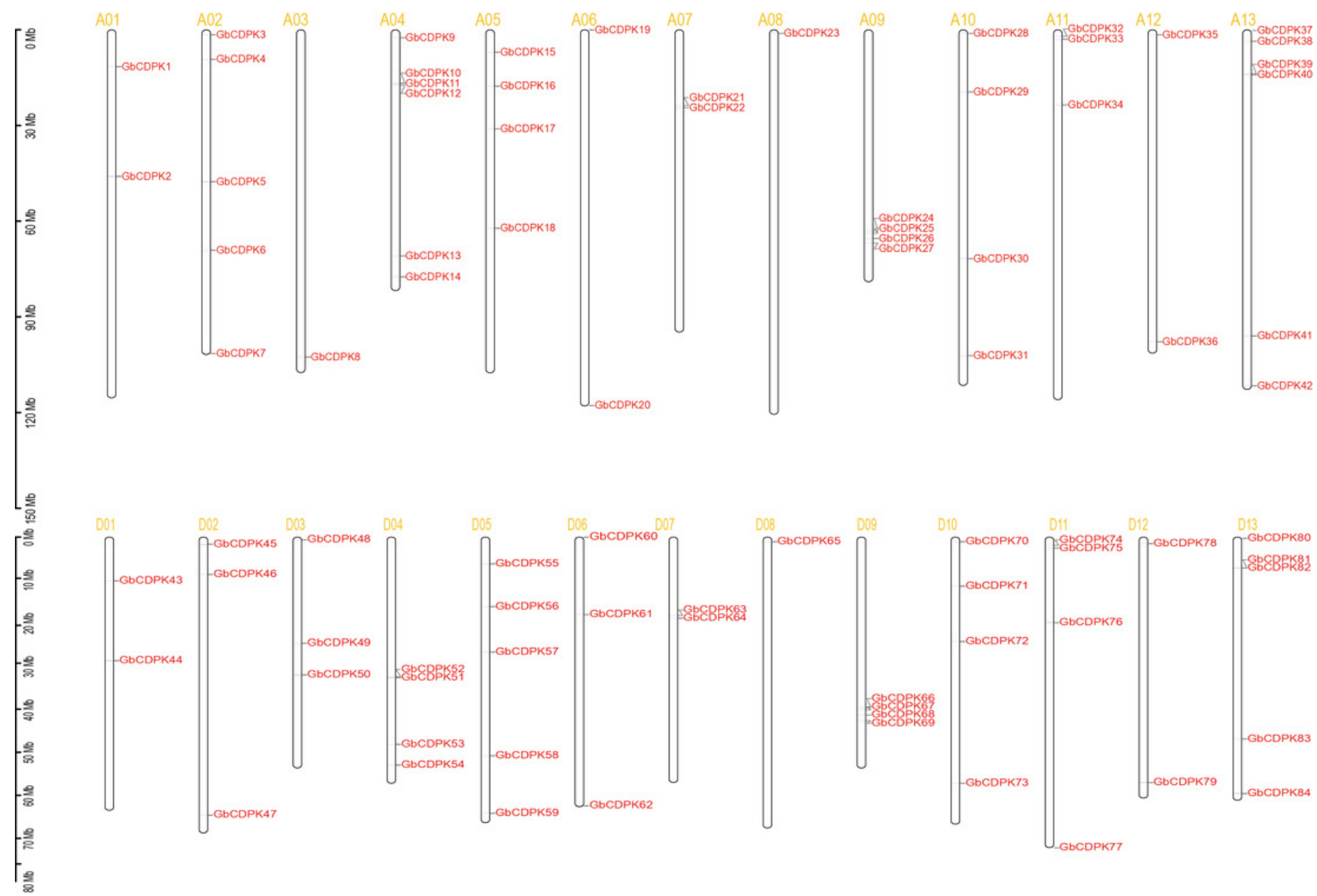


Figure 3

Whole genome duplication distribution of CDPK genes in G. barbadense

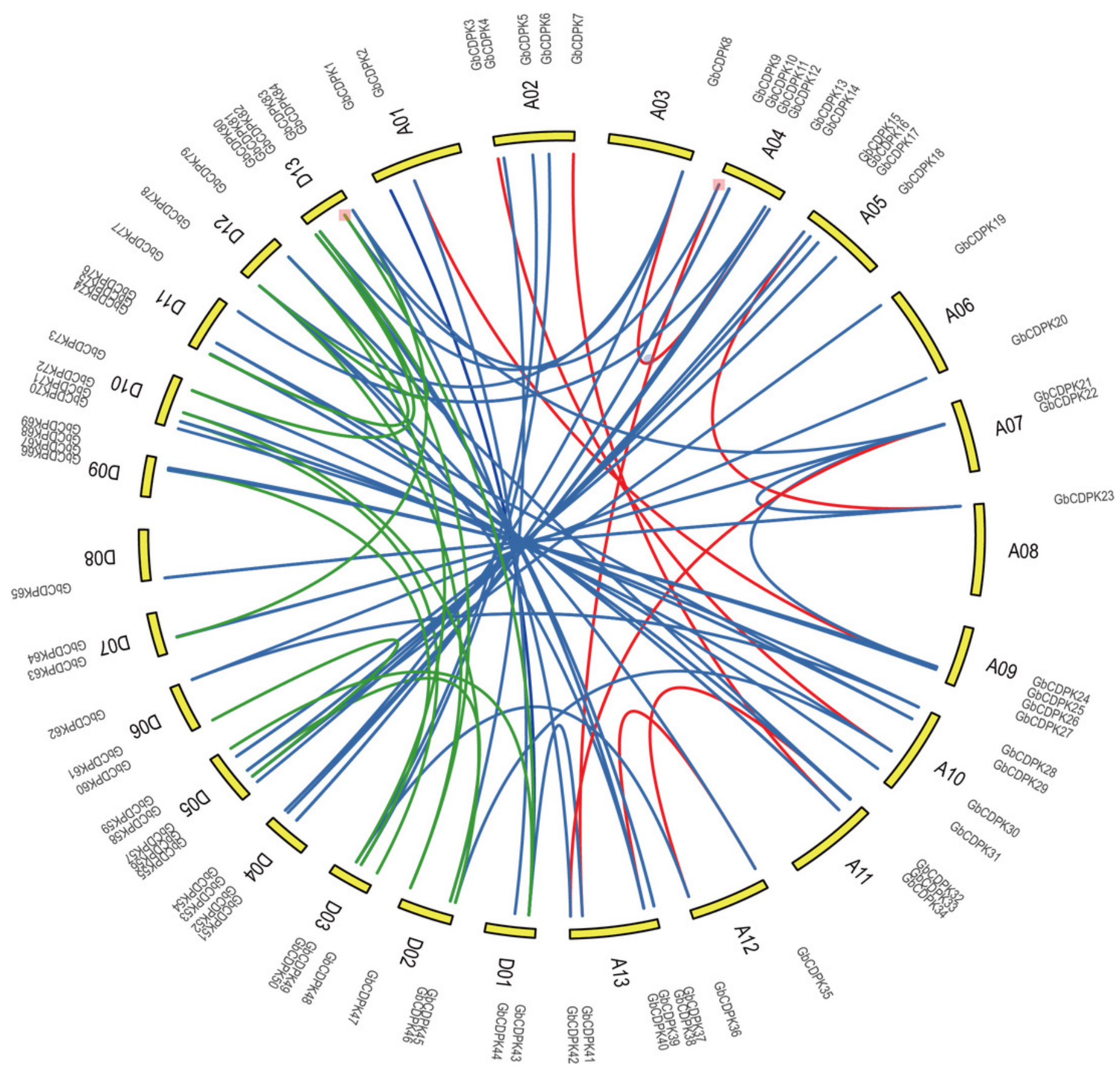


Figure 4

Phylogenetic tree, conservative motif and gene structure in G. barbadense 
A

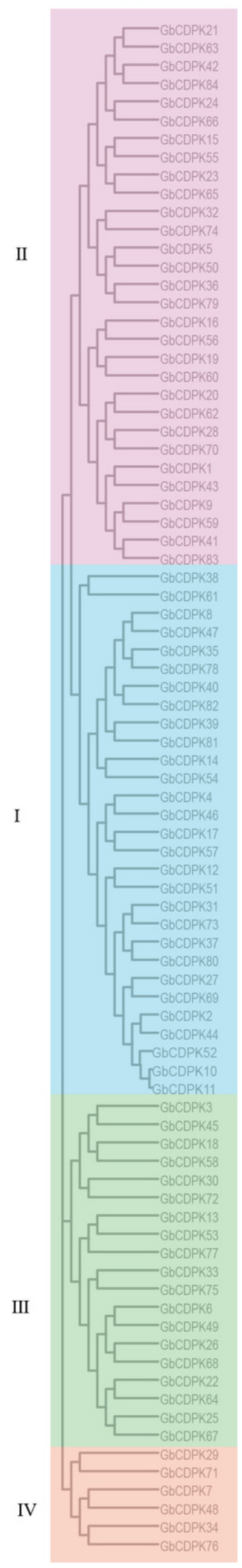

B

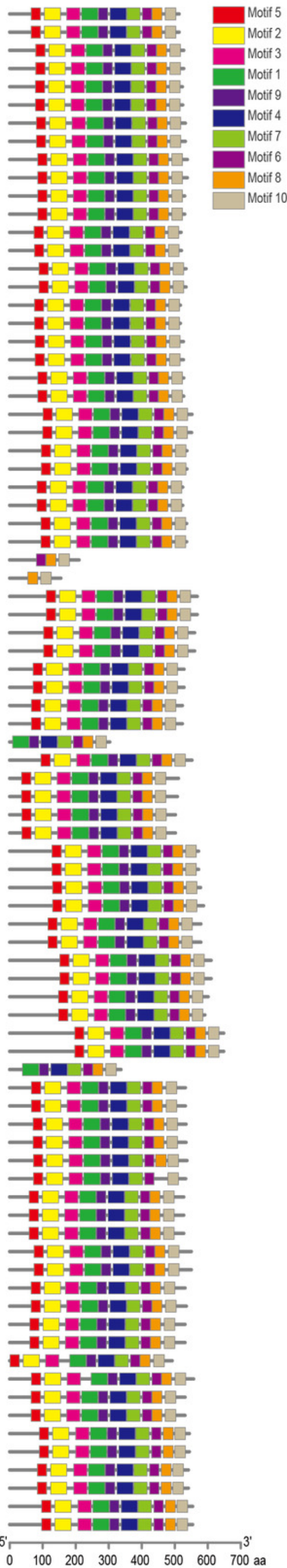

C

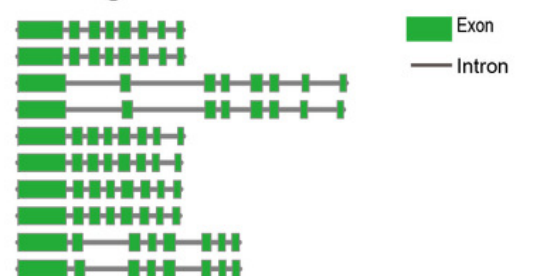

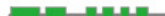

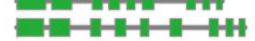

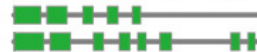

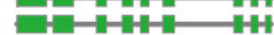

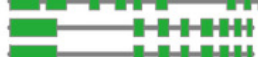

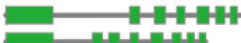

-

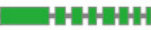

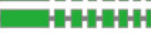

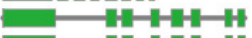

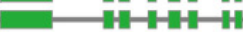

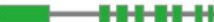

-

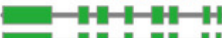

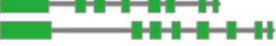

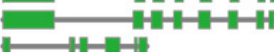

1-

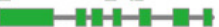

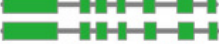

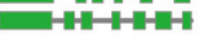

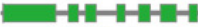

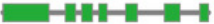

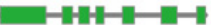

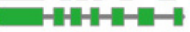

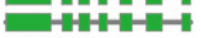

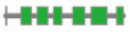

-

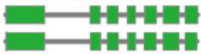

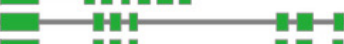

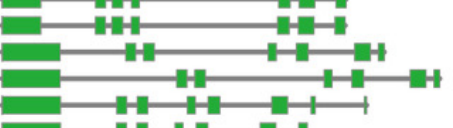

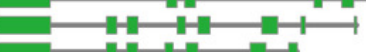

$\longrightarrow$

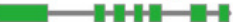

$\longrightarrow-4+4-4$

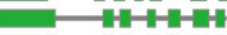

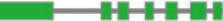

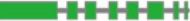

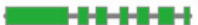

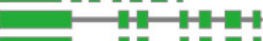

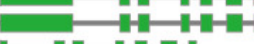

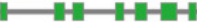

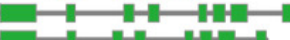

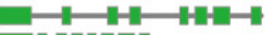

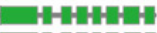

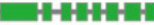

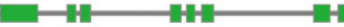
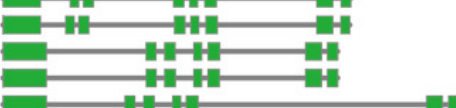

(1)

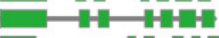

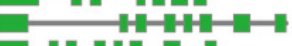

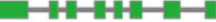

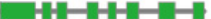

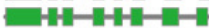

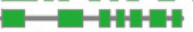

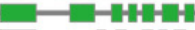

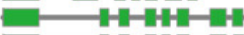

$\longrightarrow$

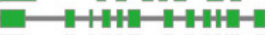

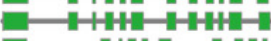

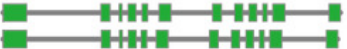

-

$-\mathrm{HH}=\mathrm{HHH}$

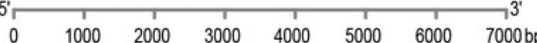

Peer) reviewing PDF | (2021:10:67134:2:1:NEW 9 Jan 2022) 
Figure 5

\section{Expression profile in different tissues and abiotic stress in GbCDPK}

A

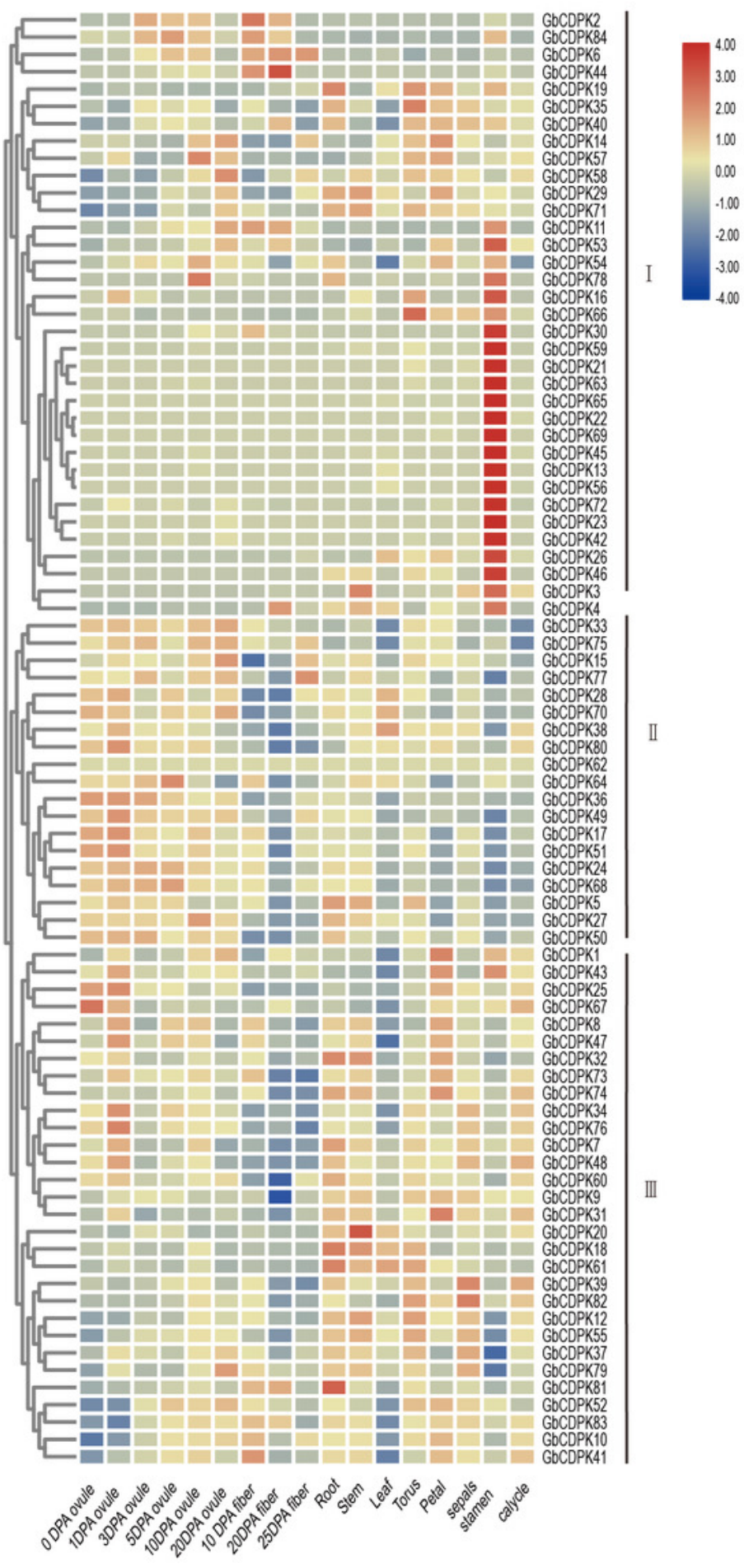

B

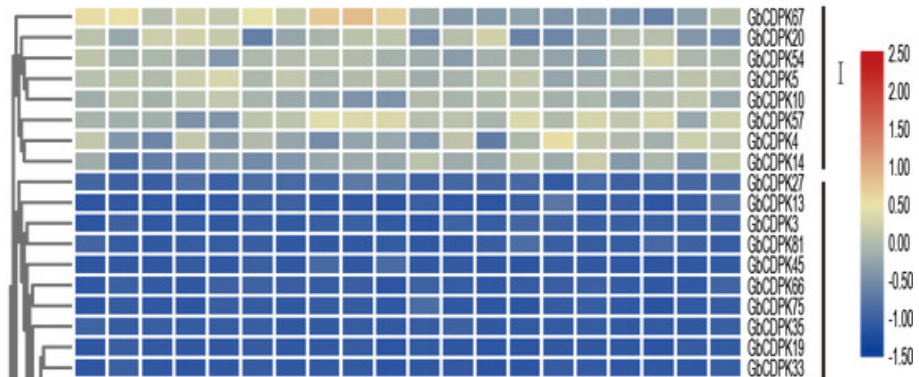

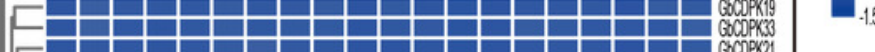

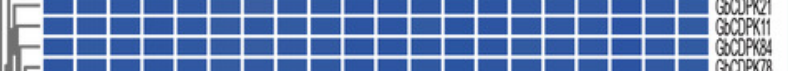

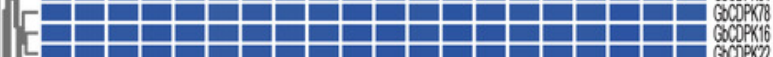

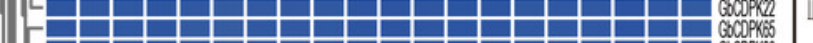

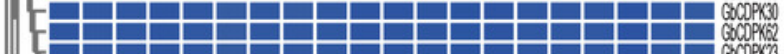

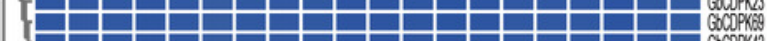

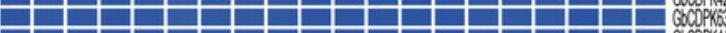

-

-

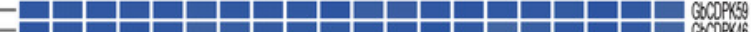

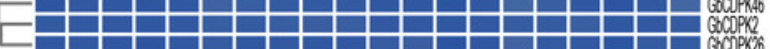

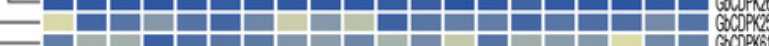

[\begin{tabular}{lllllllll}
\hline \\
\hline
\end{tabular}

\begin{tabular}{c|c|c|c|c|c|c|c|c|c|c|c|}
\hline \\
\hline
\end{tabular}

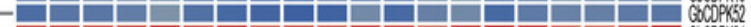

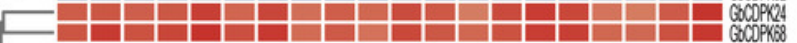

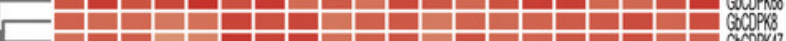

[-

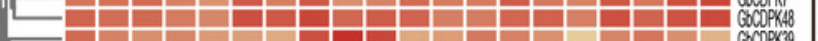

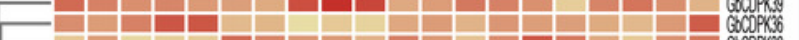

F

C+

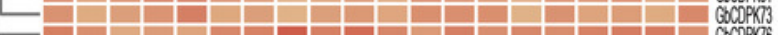

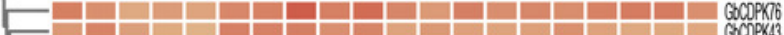

\begin{tabular}{c|c|c|c|c|c|c|c|c|c|}
\hline \\
\hline
\end{tabular}

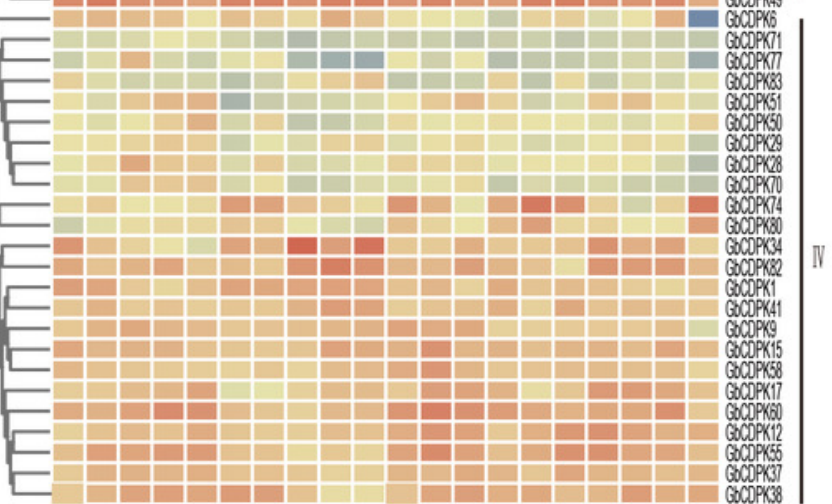

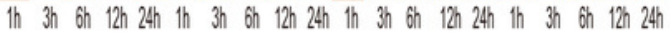


Figure 6

\section{Abiotic stress expression of CDPK genes}
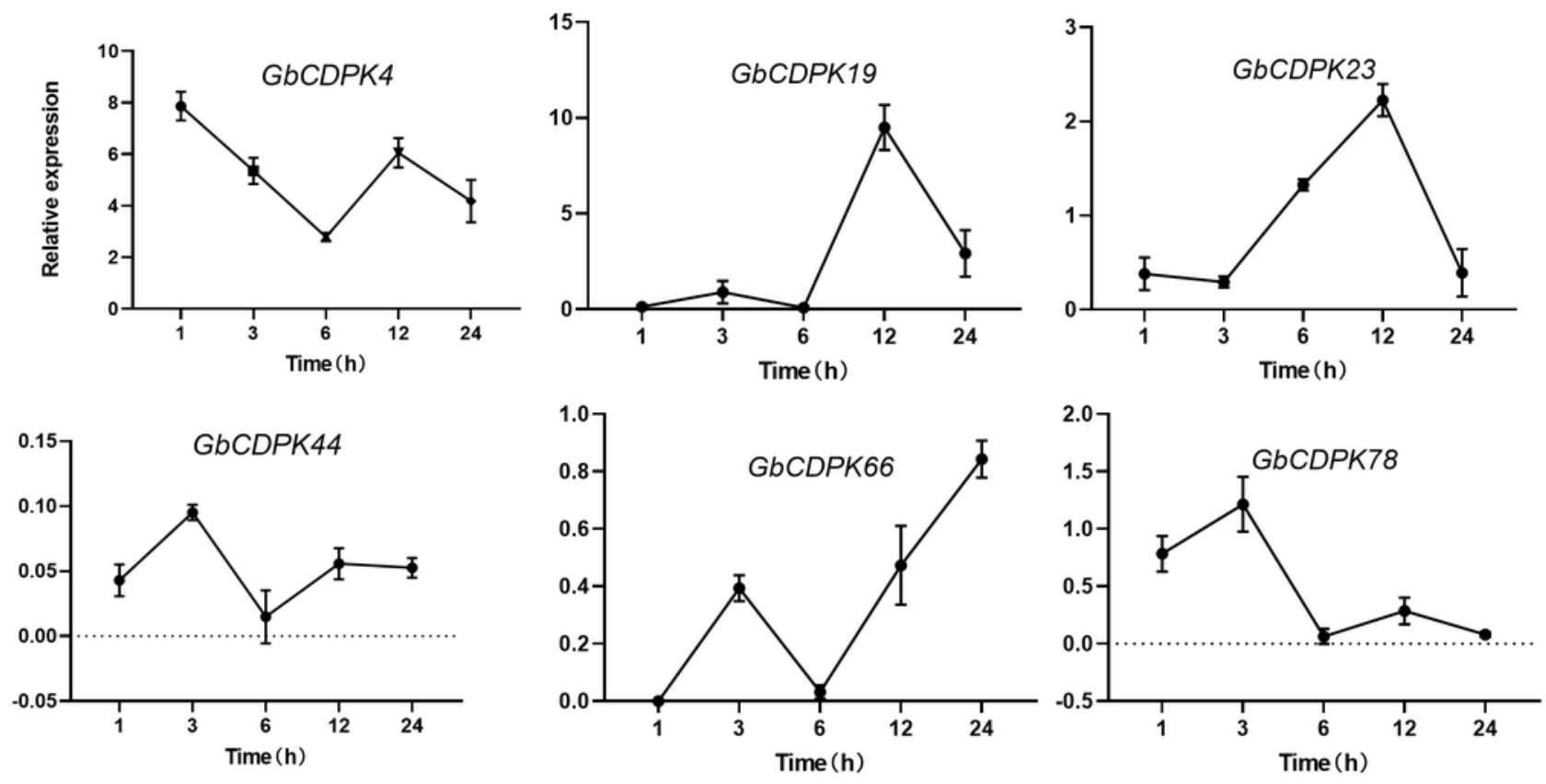
Figure 7

Functional analysis of GbCDPKs in response to drought stress

A

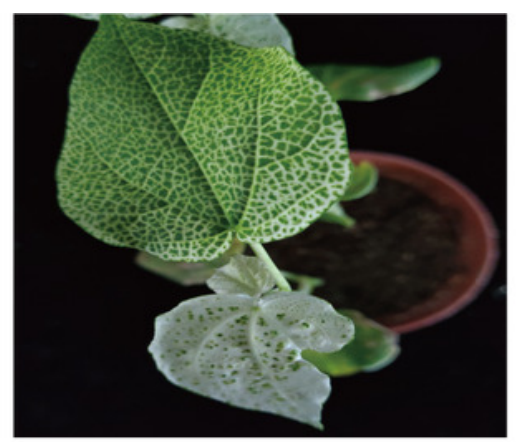

B

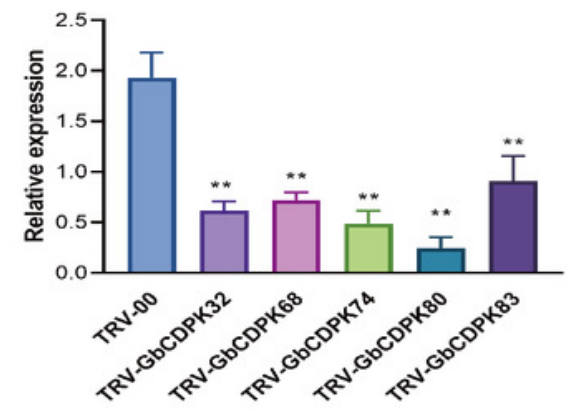

C

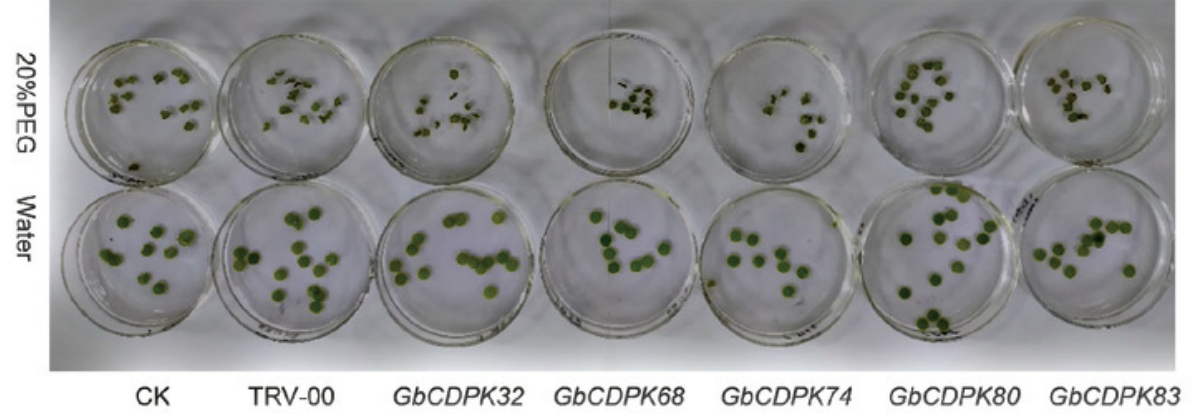

-TRV-00 TRV-GbCDPK32 - TRV-GbCDPK68 -TRV-GbCDPK74 =TRV-GbCDPK80 = TRV-GbCDPK83

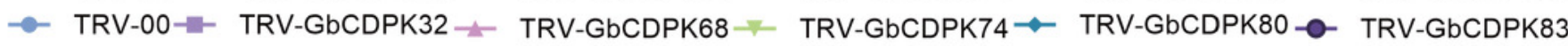

D

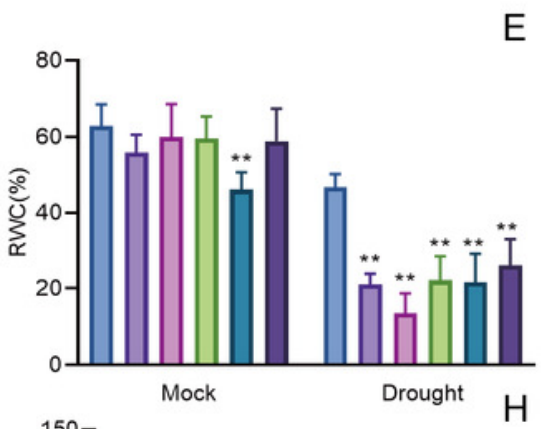

$E$

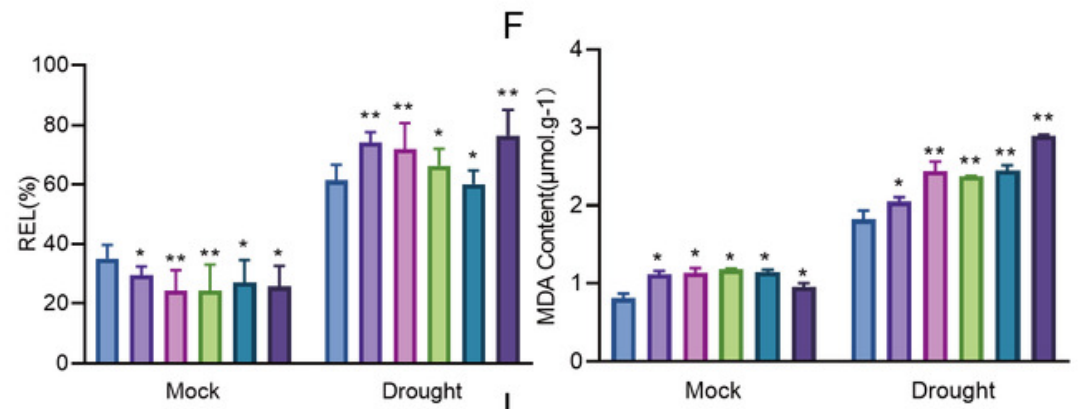

G
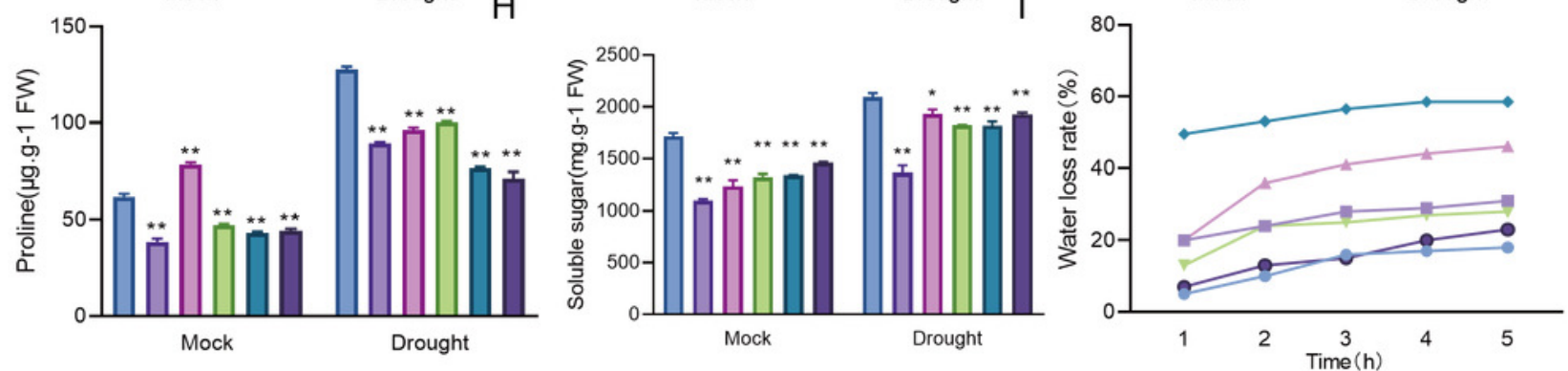